\title{
Weighting Methods for Multi-Criteria Decision Making Technique
}

\author{
ODU, G.O. \\ Department of Mechanical Engineering, Faculty of Engineering, Delta State University, Abraka, Oleh Campus, 331107, Nigeria. \\ Email:odugodwin@gmail.com
}

\begin{abstract}
Determining criteria weights is a problem that arises frequently in many multi-criteria decision-making (MCDM) techniques. Taking into account the fact that the weights of criteria can significantly influence the outcome of the decision-making process, it is important to pay particular attention to the objectivity factors of criteria weights. This paper provides an overview of different weighting methods applicable to multi-criteria optimization techniques. There are a lot of concept been reported from the literature that are very useful in solving multicriteria problems. The present work emphasized on the use of these weighting methods in determining the criteria preference of each criterion to bring about desirable properties and in order to establish and satisfy a multiple measure of performance across all the criteria selected by identifying the best options possible. And from the results, it shows that subjective weighting methods are easy and straight forward in terms of their computations than the objective weighting methods which derived their information from each criterion by adopting a mathematical function to determine the weights without the decision-maker's input,. This can be seen from the pairwise comparison which gives an internal storage and random access memory of a smart phone a weight value of 0.33 and 0.22 respectively as they have the highest criteria weights.
\end{abstract}

\section{DOI: $\underline{\text { https://dx.doi.org/10.4314/jasem.v23i8.7 }}$}

Copyright: Copyright (C) 2019 Odu. This is an open access article distributed under the Creative Commons Attribution License (CCL), which permits unrestricted use, distribution, and reproduction in any medium, provided the original work is properly cited.

Dates: Received: 20 May 2019; Revised: 27 July 2019; Accepted 31 July 2019

KEYWORDS: Multi-criteria, Decision-making, Relative importance, Alternative, Criteria

In most multi-criteria decision making (MCDM) models, assigning weights to criteria is an important step that needs to be reexamined. Though, determining the weights of criteria is one of the key problems that arise in multi-criteria decision making (Dragan et al., 2018). There are various weighting methods that have been proposed in literature and applied for solving different MCDM problems such as goal programming, Analytic Hierarchy Process (AHP), weighted score method, VIKOR, TOPSIS, etc. These weighting methods are classified in different ways: Direct criteria weighting methods (scaling, ranking-weight, point allocation procedures and an indirect approach (weight derived from theories and mathematical model). In practice, it is difficult even for a single decision maker to supply numerical relative weights of different decision criteria. Naturally, obtaining criteria weights from several decision makers is more difficult. Quite often, decision makers are much more comfortable in simply assigning ordinary ranks to the different criteria under consideration. In such cases, relative criteria weights can be derived from criteria ranks supplied by decision makers. The decision for selecting an appropriate weighting method is a difficult task in solving a multi-criteria decision problem. Several researchers have dismissed the difficulty in measuring the criteria weights and assume that the importance of criteria weights is conversant with all decision makers (Zardari et al., 2015). However, the validity of criteria weights obtained from different weighting methods cannot be ignored so as not to avoid any misuse of the MCDM models and getting reliable model results. MCDM methods can help to improve the quality of decisions by making the decision making process more explicit, rational, and efficient (Arvind and Janpriy, 2018). The author pointed out that multi criteria decision making (MCDM) is regarded as a main part of modern decision science and operational research, which contains multiple decision criteria and multiple decision alternatives.

Several researchers have come up with different methods of determining the criteria weights of a multicriteria decision making problem (Ginevicius and Podvezko 2005; Diakoulaki et al, 1995, Aldian and Taylor 2005; Dragan et al., 2018). Amongst is the weighted sum method (WSM) known to be the earliest and probably the most widely used method. The WSM was later modified to weighted product method (WPM) in order to overcome some gaps associated with it. In 1977, Saaty proposed the analytical hierarchy process (AHP) and it has recently become one of the popular methods in most MCDM techniques. Nowadays, modification to the AHP is considered to be prevalent than the original approach, e.g., the fuzzy AHP method. However, some challenges surrounds the theoretical 
basis of the method, it is easy to use and gives results that are expected to the users. Despite its ease of use, the procedure for processing information obtained from the decision maker is difficult to ascertain. This makes the method less suitable for situations with many stakeholders. Moreover for AHP, the number of pairwise comparisons increases rapidly with the number of criteria which makes it cumbersome. Other commonly used methods are the ELETRE, VIKOR and the TOPSIS methods. The ELETRE (Elimination and Choice Translating Reality) was first introduced in 1968 by Bernard Roy to deal with outranking relations which deals with problem of ranking alternatives from the best to worst by using pairwise comparisons among alternatives considering each criterion separately. Though with the outranking relationship, decision maker may still take the risk of regarding one of the alternatives better than the other. This means that the decision maker has a weak or strict preference for one of the alternatives and sometimes unable to identify the most preferred alternatives because of their difficulty to determine the alternative over the other. However, this method has the ability of eliminating less favourable alternatives and is convenient when there are decision problems that require fewer criteria with a large number of alternatives. In addition, ELECTRE method consists of a pairwise comparison of alternatives, based on the degree to which evaluations of the alternatives and the preference weights confirm or contradict the pairwise dominance relationship between alternatives. It examines both the degree to which the preference weights are in agreement with pairwise dominance relationships and the degree to which weighted evaluations differ from each other. These stages are based on a "concordance and discordance" set; hence, this method is also called concordance analysis. The VIKOR method is a multi-criteria decision making (MCDM) method. It was originally developed by Serafim Opricovic to solve decision problems with conflicting and non-commensurable criteria, assuming that compromise is acceptable for conflict resolution (Arvind and Janpriy, 2018). This method focuses on ranking and selecting from a set of alternatives and determines the compromise solution closest to the ideal solution. Chatterjee et al. (2012) proposed decision-making methodology for material selection using compromise ranking method known as Vlse Kriterijumska Optimizacija Kompromisno Resenje' (VIKOR), which means multi-criteria optimization and compromise solution. The TOPSIS method is based on technique of ranking preferences by similarity to the ideal solution (TOPSIS) to aid in material selection process proposed by Hwang and Yoon in 1980 (Xu, 2007). According to this technique, the best alternative would be the one that is closest to the positive-ideal solution and farthest from the negative ideal solution. The primary concept of TOPSIS approach is that the most preferred alternative should not only have the shortest distance from the positive ideal solution, but also have the farthest distance from the negative ideal solution (Vinodh et al., 2014). The Euclidean distance approach was proposed to evaluate the relative closeness of the alternatives to the ideal solution. Thus, the order of preference of the alternatives can be obtained by a series of comparisons of these relative distances. The entropy method is the method used for assessing the weight in a given problem because with this method, the decision matrix for a set of candidate materials contains a certain amount of information. The entropy works based on a predefined decision matrix. Using the entropy method, it is possible to combine the material designer's priorities with that of the sensitivity analysis. The TOPSIS method first converts the various criteria dimensions into non-dimensional criteria. When the designer finds no reason to give preference to one criterion over another, the principle of insufficient reason (Star and Greenwood 1977) suggests that each one should be equally preferred. However, some modification of TOPSIS has been proposed by Jahanshahloo (2006), Liu and Zeng, (2008), Rao and Davim, (2008), and Rao and Patel (2011).

Weights assigned to criteria in multi-criteria evaluation has both qualitative and quantitative data so as to make sure that the weight is taking into account for better and more accurate decision making. However, assigning weights using qualitative data to criteria can be influenced by decision maker preference, and due to this set back, Saaty (1977) proposed a numerical scale of "1-9" in order to transform qualitative data into quantitative by describing ' 1 ' as equal importance and '9' as extreme importance (Abel et al., 2018). Weights classification can also be grouped into three categories: Subjective, objective and integrated or combined weighting approach (Ginevicius and Podvezko 2005).

Subjective weight determination is based on expert opinion, and in order to get the subjective judgments, analyst normally presents the decision makers a set of questions in the process. However, subjective criteria weight determination is often time consuming especially when there is no agreement between decision makers of the problem under consideration. Example of the subjective weighting method is the Analytical Hierarchy analysis (AHP), Olson (2008) wrote on the subjectivity in multiple criteria decision analysis; he argued that judgment is at the heart of human decisionmaking and, therefore, considered judgment to be subjective. If a decision were to be made objectively, one should simply adopt the "decision support" view 
that human decision-makers should be entrusted with the final decision, and that every model is imperfect. Models do not include all factors. Even the most careful attempts at objective measurement will inevitably involve some inaccuracy. The author also pointed out that we must accredit our own judgment as the paramount arbiter. In the objective weighting methods, criteria weights are derived from information gathered in each criterion through mathematical models without any consideration of the decision maker's intervention (Aldian and Taylor, 2005). The integrated weighting approach is a weighting method based on the combination of subjective weighting and objective weighting methods. It focuses on the principle of integrating the subjective weights based on expert's opinion due to his/her knowledge and experience in the relevant field and the information gathered from the criteria data in a mathematical form (objective weighting method). In the subsequent section, the mathematical function and specific examples of each of these methods will be illustrated and evaluated.

Now, let us look at some of the most common subjective weights that have been used in previous MCDM studies are shown in Table 1.

\begin{tabular}{|c|c|c|}
\hline Subjective weighting methods & $\begin{array}{l}\text { Weighting methods } \\
\text { Objective weighting methods }\end{array}$ & Integrated weighting methods \\
\hline Point allocation & Entropy method & Multiplication synthesis \\
\hline Direct rating & $\begin{array}{l}\text { Criteria Importance Through Inter-criteria } \\
\text { Correlation (CRITIC) }\end{array}$ & Additive synthesis \\
\hline Ranking method & Mean weight & $\begin{array}{l}\text { Optimal weighting based on sum of } \\
\text { squares }\end{array}$ \\
\hline Pairwise comparison (AHP) & Standard deviation & $\begin{array}{l}\text { Optimal weighting based on relational } \\
\text { coefficient of graduation }\end{array}$ \\
\hline Ratio method & Statistical variance procedure & \\
\hline Swing method & Ideal point method & \\
\hline \multicolumn{3}{|l|}{ Delphi method } \\
\hline \multicolumn{3}{|l|}{ Nominal group technique } \\
\hline \multicolumn{3}{|l|}{ Simple Multi-attribute Ranking } \\
\hline Technique (SMART) & & \\
\hline
\end{tabular}

Subjective weighting methods: The most commonly used subjective weighting methods are listed in Table 1 are as follows:

(1) The point allocation method: This is one of the simplest methods used to determine criteria weights according to the priority of criteria, a decision-maker allocates a certain number of points to each criterion. The more points a criterion receives, the greater its relative importance (Golaszewski et al., 2012). In this scenario, the decision maker is asked to allocate 100 points across the criteria under consideration. The total of all criterion weights must sum up to 100 . This method is easy to normalize. However, the weights obtained from the use of point allocation method are not very precise, and the method becomes more difficult as the number of criteria increases to 6 or more. For example, consider five key quality characteristics of smart phone one should look out for: cost, display resolution, battery life, random memory, and internal storage.

Table 2: Smart phone criteria weights using point allocation method

\begin{tabular}{lll}
\hline S/N & Criteria & Weights \\
\hline 1 & Cost & 10 \\
2 & Display Resolution & 35 \\
3 & Battery Life & 15 \\
4 & Random Access Memory (RAM) & 25 \\
5 & Internal Storage & 15 \\
& Total & 100 \\
\hline
\end{tabular}

(2) The direct Rating method: The direct rating method is a type of approach in which the decisionmaker first ranks all the criteria according to their importance. The rating does not constrain the decision maker's responses as the fixed point scoring methods does. It is possible to alter the importance of one criterion without adjusting the weight of another (Arbel, 1989).

(3) The pairwise comparisons: This method is used for analyzing multiple populations in pairs to determine whether they are significantly different from one another. It can also put as a method where the decision-maker compares each criterion with others and determines the level of preferences for each pair of such criteria. The use of ordinal scale $(1-9)$ is adopted to help in determining the preference value of one criterion against the other. And one of the most commonly applied methods based on pairwise comparisons is the Analytical Hierarchy process (AHP) method. The number of comparisons can be determined by

$$
c_{p}=\frac{n(n-1)}{2}
$$


Where $c_{p}=$ the number of comparisons: $\mathrm{n}=$ the number of criteria

Determining the criteria weights based on pairwise comparisons method has three main steps and can be implemented as follows. The first step is to develop a matrix by comparing the criteria as shown in Table 4 . Intensity values are used to fill the matrix, such as $(1,3,5,7,9)$ representing equal importance, moderate importance of one over the other, strong importance, very strong importance, extreme importance respectively,. While the ordinal scale of 2,4,6 and 8 are intermediate values or when compromise is needed and can be represented as follows: equally to moderately preferred -2 ; moderately to strongly preferred - 4; strongly to very strong importance -6 ; and very strong to extremely strong importance -8 .

The diagonal in the matrix is always 1 and the lower left values are inverse values if activity $i$ has one of the above numbers assigned to it when compared with activity $j$, then $j$ has the reciprocal value when compared with $i$. To fill the lower triangular matrix, we use the reciprocal values of the upper diagonal. Thus we have complete comparison matrix.

The second step is to calculate the criteria weight, which is also known as priority value or the principal eigenvector. This is done by using either of the following methods:

Method 1: By summing the values in each column, dividing each element by the column total, and dividing the sum of the normalized scores for each row by the number of criteria as shown in the given example (Table 4). The calculation for the priority value of the first row in the matrix is given as: summation for the first column total is 22 , and the remaining four columns gives $4.33,3.44,5.70$, and 5.25. Therefore, the priority value for the first row is gives

$\left(\frac{1}{22}+\frac{1 / 3}{4.33}+\frac{1 / 9}{3.44}+\frac{1 / 5}{5.70}+\frac{1 / 4}{5.25}\right) / 5=0.05$

Method 2: Multiplying together the entries in each row of the matrix and then taking the nth root of that product gives a very good approximation. The nth roots are summed and that sum is used to normalize the eigenvector elements to add to 1.00 . For the example, in Table 4, the number of criteria or attribute is five, which is the fifth root for the first row is 0.283 and that is divided by 5.88 to give 0.05 as the first criteria weight.

The third step is to estimate the consistency for sensitivity analysis known as consistency ratio (CR). If the consistency ratio is less than 0.1 , then the ratio indicates a reasonable level of consistency in the pairwise comparisons, but once the $\mathrm{CR}$ is greater than 0.1 , it shows that the pairwise comparisons are inconsistent in judgment. Sensitivity analysis can be useful in providing information as to the robustness of any decision. In order to compute the consistency ratio, the following procedure needs to be followed: (a) multiply each value in the first row of the pairwise comparisons matrix by corresponding criteria weight or eigenvector to obtain a new vector. For example,

$1 \times 0.005+\frac{1}{3} x 0.20+\frac{1}{9} x 0.33+\frac{1}{5} x 0.22+\frac{1}{4} \times 0.2=0.246$

(b) Repeat step (a) for remaining columns, that is, the remaining four rows give $1.100,1.840,1.179$, and 1.040 as the five elements of $\lambda_{\max }$. (c) Divide each elements of the vector of weighted sums obtained in step $\mathrm{a}-\mathrm{b}$ by the corresponding priority value. Each component of $(0.246,1.100,1.840,1.179,1.040)$ by the corresponding criteria weight. This gives $\frac{0.246}{0.05}=4.926$ for the first row, other values are 5.500, 5.576, 5.359, and 5.200. (d) Then compute the average of the values found in step c, let $\lambda_{\max }$ be the average. The mean of these values is 5.312 , which is the estimate of the $\lambda_{\text {max }}$. If any of the estimates for $\lambda_{\text {max }}$ turns out to be less than $n$, or 5 in this case, there has been an error in the calculation. (e) Compute the consistency index $(\mathrm{CI})$, which is defined as

$$
C I=\frac{\lambda_{\text {max }}-n}{n-1}
$$

(f) Using random judgments from Table 3 which was derived from Saaty's book, in which a set of judgments for the corresponding value from large samples of matrices for the computation of consistency ratio, in which the upper row is the order of the random matrix, and the lower row is the corresponding index of the consistency for random judgments referred to as random index

Table 3: Random Index

\begin{tabular}{|c|c|c|c|c|c|c|c|c|c|c|c|c|c|c|c|}
\hline $\begin{array}{l}\text { Order } \\
\text { RI }\end{array}$ & 19 & $\begin{array}{l}2 \\
000\end{array}$ & $\begin{array}{l}3 \\
0.58\end{array}$ & $\begin{array}{l}4 \\
090\end{array}$ & $\begin{array}{l}5 \\
112\end{array}$ & $\begin{array}{l}6 \\
124\end{array}$ & $\begin{array}{l}7 \\
132\end{array}$ & $\begin{array}{l}8 \\
141\end{array}$ & $\begin{array}{l}9 \\
145\end{array}$ & $\begin{array}{l}10 \\
149\end{array}$ & $\begin{array}{l}11 \\
151\end{array}$ & $\begin{array}{l}12 \\
154\end{array}$ & $\begin{array}{l}13 \\
156\end{array}$ & $\begin{array}{l}14 \\
157\end{array}$ & $\begin{array}{l}15 \\
159\end{array}$ \\
\hline & & & & & & 1.24 & $\frac{1.3}{\text { irce }: S}$ & & 1.45 & & & & 1.56 & 1.57 & 1.59 \\
\hline
\end{tabular}


(g) Therefore, the consistency ratio, $\mathrm{CR}$ is

$C R=\frac{C I}{R I}$; That means $C R=\frac{0.078}{1.12}=0.07$

Accept the matrix if consistency ratio is less than 0.1 or $10 \%$. Higher numbers indicates that the comparisons are less consistent, while smaller numbers mean comparison are more consistent, CR above 0.1 or $10 \%$ indicates that the pairwise comparisons should be revisited or reversed (Setiawan et al., 2014).

\begin{tabular}{|c|c|c|c|c|c|c|c|c|c|c|}
\hline & $\mathrm{RD}$ & $\mathrm{BL}$ & RAM & INS & $\mathrm{C}$ & $\begin{array}{l}\text { nth root of } \\
\text { product } \\
\text { values }\end{array}$ & Priority & $\mathrm{CI}$ & RI & $\mathrm{CR}$ \\
\hline RD & 1 & $1 / 3$ & $1 / 9$ & $1 / 5$ & $1 / 4$ & 0.283 & 0.05 & 0.08 & 1.12 & 0.07 \\
\hline BL & 3 & 1 & 1 & 1 & 1 & 1.246 & 0.20 & & & \\
\hline RAM & 9 & 1 & 1 & 3 & 1 & 1.933 & 0.33 & & & \\
\hline INS & 5 & 1 & $1 / 3$ & 1 & 2 & 1.270 & 0.22 & & & \\
\hline Cost (C) & 4 & 1 & 1 & $1 / 2$ & 1 & 1.149 & 0.20 & & & \\
\hline Totals & & & & & & 5.880 & 1.00 & & & \\
\hline
\end{tabular}

(4) Ranking method: This is one of the simplest approaches to assign criteria weights. The criteria are usually ranked from best to worst importance. There are three approaches to calculate weights using the ranking method. They include rank sum, the rank exponent and rank reciprocal. In the rank sum, the weights are computed from the individual ranks normalized by dividing the sum of the ranks. The formula for rank sum determination can be expressed as (Raszkowska, 2013):

$$
w_{j}(R S)=\frac{n-p_{j}+1}{\sum_{k=1}^{n} n-p_{k}+1}
$$

Where $p_{j}$ is the rank of the $j$-th criterion, $j=1,2, n$

The rank exponent weight (RE) method is a similar to the rank sum method except that the value is raised to an exponential of a parameter $p$ which may be estimated by a decision maker as a result of the most important criterion. The formula for the rank exponent method is given as (Raszkowska, 2013):

$$
w_{j}(R E)=\frac{\left(n-p_{j}+1\right)^{p}}{\sum_{k=1}^{n}\left(n-p_{k}+1\right)^{p}}
$$

Where $p_{j}$ is the rank of the $j$-th criterion, and $p$ is the parameter describing the weights,

$j=1,2, \ldots, n$

The reciprocal (or inverse) weights (RR) method uses the normalized reciprocal of the criterion rank. This can be expressed as (Raszkowska, 2013):

$$
w_{j}(R R)=\frac{1 / p_{j}}{\sum_{k=1}^{n}\left(1 / p_{k}\right)}
$$

Where $p_{j}$ is the rank of the $j$-th criterion, $j=1,2, n$

For clearer understanding, an example on the computation of weights using rank sum, rank exponent and rank reciprocal method see Table 5. These methods are actually not appropriate for a large number of criteria due to the difficulty of straight ranking. However, these techniques should be regarded as weight approximation method only

\begin{tabular}{|c|c|c|c|c|c|c|c|}
\hline \multirow[b]{2}{*}{ Criteria } & \multirow[b]{2}{*}{$\begin{array}{l}\text { Str. } \\
\text { Rank }\end{array}$} & \multicolumn{2}{|c|}{$\begin{array}{l}\text { Rank Sum } \\
n-p_{j}+1\end{array}$} & \multicolumn{2}{|c|}{$\begin{array}{c}\text { Rank exponent } \\
\left(n-p_{j}+1\right)^{p} \\
p=2\end{array}$} & \multicolumn{2}{|c|}{$\begin{array}{c}\text { Rank reciprocal } \\
1 / p_{j}\end{array}$} \\
\hline & & Weight & Normalized & weight & normalized & weight & normalized \\
\hline Physics & 5 & 2 & 0.065 & 4 & 0.044 & 0.20 & 0.082 \\
\hline Chemistry & 2 & 5 & 0.161 & 25 & 0.275 & 0.50 & 0.204 \\
\hline Biology & 4 & 3 & 0.097 & 9 & 0.099 & 0.25 & 0.102 \\
\hline Mathematics & 6 & 1 & 0.032 & 1 & 0.011 & 0.17 & 0.068 \\
\hline English & 3 & 4 & 0.129 & 16 & 0.176 & 0.33 & 0.136 \\
\hline \multirow[t]{2}{*}{ Agric. Sc. } & 1 & 6 & 0.194 & 36 & 0.396 & 1.00 & 0.408 \\
\hline & Total & 31 & & 91 & & 2.45 & \\
\hline
\end{tabular}
because of its simplicity and gives an easy check of criteria weights.

Note: 1 is the most important criterion, and 6- is the least important out of the six criteria using the straight rank first 
(5) Ratio weighting method: The ratio method is one of the subjective weighting methods that requires the input of the decision makers to rank the relevant criteria according to their importance. Here, the least important criterion is assigned the value of 10 , and the other criteria are assigned multiples of 10 . The resulting weights are then normalized to sum to one.

(6) Swing weighting method: In the swing weighting method, the decision maker is asked to select an alternative with the worst outcome and picks the criteria whose performance is likely to change (or swing) from its worst to the best. The criterion with the most preferred swing is given higher weight, e.g., 100 points value. Next, the criteria whose performance the decision maker would like to change from its worst to the best level is selected again and a value between 0 and 100 representing its relative importance regarding the most important criteria is provided. Then obtain the average normalized weights and normalized weights interval (Parnell and Trainor, 2009). A typical template for swing weight matrix is given in Table 6 . The first step is to create a matrix in which the top defines the values in terms of the relative importance while the left hand side represents the range of variation values. Assign a value measure that is most preferred to the decision and at the same time has a large variation to the upper left of the matrix labeled cell ' $R$ '. And a value measure that has the worst preferred importance and has the smallest variation in its scale is placed to the lower right of the matrix labeled cell ' $Z$ '.

\begin{tabular}{lllll}
\multicolumn{3}{c}{ Table 6: Swing weight matrix template } \\
\hline & & \multicolumn{3}{c}{ Importance of the value } \\
measure to the decision makers \\
\cline { 3 - 5 } & & High & Medium & Low \\
\hline Variation & High & R & S & T \\
of Scale & Medium & U & V & W \\
& Low & X & Y & Z \\
\hline
\end{tabular}

However, the consistency rule for swing weight method is very important and necessary to ensure consistency of the weights assigned to individual cells of the matrix. And for the consistency rules to hold, the following conditions or relationship of nonnormalized swing weights must be followed:

(a) $\quad C_{R}>C_{i}$ for all value of $i$ in all other cell

(b) $C_{U}>C_{X}, C_{V}, C_{Y}, C_{W}, C_{Z}$

(c) $C_{S}>C_{V}, C_{T}, C_{Y}, C_{W}, C_{Z}$

(d) $C_{X}>C_{Y}, C_{Z}$

(e) $C_{V}>C_{Y}, C_{W}, C_{Z}$

(f) $\quad C_{T}>C_{W}, C_{Z}$ (g) $\quad C_{Y}>C_{Z}$

(h) $\quad C_{W}>C_{Z}$

In assigning swing weights, the stakeholders need to make compromise between level of importance and level of variation in measure scale. This is done by allowing the stakeholders to assign arbitrary large weight to the top left hand side of the matrix, for example, 1000 or 100 as shown in Table 7

$C_{R}=100$ ) and the weight of the lowest importance in cell $\mathrm{Z}\left(C_{Z}=1\right)$. Then using the expression in equation (9) to calculate the normalized swing value for $i^{\text {th }}$ value.

$W_{i}=\frac{C_{i}}{\sum_{i=1}^{n} C_{i}}$

Where $C_{i}$ is the unnormalized swing weight.

Table 7: Element of the swing weight matrix

\begin{tabular}{lllll}
\hline & & \multicolumn{3}{l}{$\begin{array}{l}\text { Importance of the value } \\
\text { measure to the decision makers }\end{array}$} \\
\cline { 3 - 5 } & & High & Medium & Low \\
\hline Variation & High & 100 & 85 & 35 \\
of & Medium & 80 & 40 & 20 \\
Scale & Low & 50 & 10 & 1 \\
\hline
\end{tabular}

(7) Nominal Group Technique (NGT): Nominal group technique is a structured brainstorming technique that is used to produce a large number of ideas concerning an issue and making sure that all the group members have equal participation. Apart from the fact that the technique can be used to generate a large number of ideas, but can also be used to prioritize the ideas and more importantly, the ideas which receive majority of the votes are selected (Abdullah and Islam 2011). In NGT the weights are derived by carrying out the following steps with experience people in a group of not less than seven members.

Step 1: Silent generation of ideas in writing: All participants are given about 10 minutes to generate as many ideas as possible with respect to the issue at hand in absolute silence and done independently.

Step 2: Round-robin recording of ideas on a flip pad: In this step, each participant is asked to provide the best idea from the list generated in the first round. All ideas are written down on a flip pad or marker board, and this is carried out in subsequent in a round-robin manner until all the ideas are exhausted in the participants' list. 
Step 3: Discussion of ideas for clarification: At this step, all the ideas are being discussed for clarification. The facilitator starts from the beginning of the master list and asks the participants whether the meaning is clear to them or not. And for whatever reason any of the idea that is not clear, then it needs to be clarified by the person who provided it or by someone else.

Step 4: Voting to select the most important ideas: This last aspect of the technique is very important because all the ideas selected will be used for determining the criteria weight. The participant will be asked to select 5 most important ideas from the master list and rate them using 1 to 5 scales according to their importance. The most important idea is assigned a rating of 5 and the least receive the rating of 1 . This rating will be done by each participant and then the aggregate voting will be computed accordingly.

(8) Simple Multi-attribute Rating Technique (SMART): The SMART technique is a compensatory method of multiple criteria decision making originally developed by Edward in 1971 (Patel et al. 2017). In the SMART method, it is described as a process of rating of alternatives and weighting criteria. So, we will be looking at the weighting approach of this method. In this method, decision maker is asked to rank the criteria in terms of their importance from worst to best. The least important a criterion is assigned 10 points while the most important criterion is given 100 points with an increasing number of points are assigned to the other criteria according to their importance. The criteria weight can be calculated by normalizing the sum of the points to one.

Objective weighting methods: As mentioned earlier, the objective weighting methods are derived from information gathered from each criterion using a mathematical function to compute the weights without the interference of the decision maker. This includes entropy method, mean weight, standard deviation, statistical variance procedure, and criteria importance through inter-criteria (CRITIC).

Entropy Method: The entropy method is the method used for assessing the weight in a given problem because with this method, the decision matrix for a set of candidate materials contains a certain amount of information. The entropy works based on a predefined decision matrix. Entropy in information theory is a criterion for the amount of uncertainty represented by a discrete probability distribution, in which there is agreement that a broad distribution represents more uncertainty than does a sharply packed one (Deng et al. 2000). The entropy method for assessing the relative importance of criteria is calculated using material data for each criterion, the entropy of the set of normalized outcomes of the jth criterion is given by

$$
\begin{aligned}
& E_{j}=-\left[\sum_{i=1}^{m} p_{i j} \ln \left(p_{i j}\right)\right] / \ln (m) ; j=1,2, \\
& \ldots, n \text { and } i=1,2, \ldots, m
\end{aligned}
$$

The $p_{i j}$ form the normalized decision matrix and is given by

$$
p_{i j}=\frac{r_{i j}}{\sum_{i=1}^{m} r_{i j}} ; i=1,2, \ldots, \text { and } ; j=1 \text {, }
$$

Where $r_{i j}$ is an element of the decision matrix, $\mathrm{k}$ is a constant of the entropy equation and $E_{j}$ as the information entropy value for $j$ th criteria. Hence, the criteria weights, $w_{j}$ is obtained using the following expression.

$$
w_{j}=\frac{1-E_{j}}{\sum_{j=i}^{n}\left(1-E_{j}\right)} ; \mathrm{j}=1,2, . ., \mathrm{n}
$$

Where $\left(1-E_{j}\right)$ is the degree of diversity of the information involved in the outcomes of the jth criterion.

(1) Mean Weight $(M W)$ : The mean weight (equal importance) is mostly adopted when there is no information from decision maker or when there is no enough information available to reach a decision (Jahan et al., 2012). The mean weight is based on the assumption that all criteria are of equal importance. This can be derived by using equation (13).

$w_{j}=\frac{1}{n}$

Where $\mathrm{n}$ is the number of criteria

(2) Standard Deviation Method: The standard deviation method determines the weights of the criteria in terms of their standard deviations using the expression in equation (14) and (15) (Jahan et al., 2012).

$$
\begin{aligned}
& \sigma_{j}=\sqrt{\frac{\sum_{i=1}^{m}\left[r_{i j}-\overline{r_{j}}{ }^{2}\right.}{m}} \quad i=1, \ldots, m \\
& j=1, \ldots, n
\end{aligned}
$$

Therefore, 


$$
w_{j}=\frac{\sigma_{j}}{\sum_{j=1}^{n} \sigma_{j}}
$$

where $\sigma_{j}$ is the standard deviation for criterion $j$

4. Statistical Variance Procedure: This is another method of an objective weighting approach based on statistical variance of information as given in equation (16)

$$
\varphi_{j}=\frac{1}{n} \sum_{i=1}^{n}\left\{\left(x_{i j}-\overline{x_{i j}}\right)^{2}\right\}
$$

Where $\varphi_{j}$ is the statistical variance; $x_{i j}$ is the average value of set of data

Hence, the weight of the criteria can be obtained as in equation (17)

$$
w_{j}=\frac{\varphi_{j}}{\sum_{i=1}^{n} \varphi_{j}}
$$

(3) Criteria importance through inter-criteria (CRITIC): The criteria importance through intercriteria correlation (CRITIC) method is based on the standard deviation proposed by Diakoulaki et al. (1995) which uses correlation analysis to measure the value of each criterion. First of all, normalized the decision matrix using equation (18) and (19).

$\rho_{i j}=\frac{y_{i j}-y_{j}{ }^{\min }}{y_{j}{ }^{\max }-y_{j}^{\min }} \quad i=1, \ldots, m ; j=1, \ldots, n$

For benefit criteria

$\rho_{i j}=\frac{y_{j}^{\max }-y_{i j}}{y_{j}{ }^{\max }-y_{j}{ }^{\min }} \quad i=1, \ldots, m ; j=1, \ldots, n$

For cost criteria

Then to calculate the weight of the criteria in equation (21), we have to compute a linear correlation coefficient between the criteria values in the matrix by employing equation (20)

$$
\begin{aligned}
v_{j k} & =\frac{\sum_{i=}^{m}\left(\rho_{i j}-\overline{\rho_{j}}\right)\left(\rho_{i k}-\overline{\rho_{k}}\right)}{\sqrt{\sum_{i=1}^{m}\left(\rho_{i j}-\overline{\rho_{j}}\right)^{2} \sum_{i=1}^{m}\left(\rho_{i k}-\overline{\rho_{k}}\right)^{2}}} ; \\
j, k=1, \ldots, n &
\end{aligned}
$$

$$
w_{j}=\frac{\beta_{j}}{\sum_{k=1}^{n} \beta_{k}}
$$

Where $\beta_{j}=\sigma_{j} \sum_{k=1}^{n}\left(1-v_{j k}\right) ; j=1, \ldots, n$

Integrated weighting method: The integrated or combines weighting methods are derived from both subjective and objective information on criteria weights. The approach determines weights by solving a mathematical model and takes into consideration both subjective and objective factors. It overcomes the shortages which occur in either a subjective or an objective approach. Most times, the weights determined by subjective method are bias and neglects the objective information aspect. The judgment of the decision makers sometimes depends on the knowledge or experience and this may affect the decision process to some extent. Therefore, literature has it that none of the two approaches are perfect, and the integrated method might be the most appropriate for determining the criteria weights. There are some numbers of combinations that have been proposed and developed by scholars. Jian et al. (1999) proposed a subjective and objective integrated approach which gives ranking of alternatives that reflects both subjective considerations and objective situations. Also, Jahan et al. (2012) proposed an integrated weighting procedure which was able to strengthen the existing MCDM material selection especially when there are numerous alternatives with inter-related criteria.

Conclusion: The paper mainly aims at the various methods for determining criteria weights in MCDM by considering subjective weighting method, objective weighting method and integrated weights approach. which allows the decision maker to assess the actual performance of a particular selection process, and makes it easier to identify the difference between the subjective and objective weighting method, and the expected level of performance that intend to achieve in the future.

\section{REFERENCES}

Abdullah, M.M.B; Islam, R. (2011). Nominal Group Technique and its Applications in Managing Quality in Higher Education. Pak. J. Com. Soc. Sci. 5(1), 81-99.

Aldian, A; Taylor, M.A.P. (2005). A consistent method to determine flexible criteria weights for multicriteria transport project evaluation in developing countries. $J$. East. Asia Soc. Transport. Stud. 6: 3948-3963. 
Arbel, A. (1989). Approximate articulation of preference and priority derivation. European J. Operate. Res. 43, 317-326.

Arvind, J; Janpriy, S, (2018). A Comprehensive literature review of MCDM techniques ELECTRE, PROMETHEE, VIKOR, and TOPSIS applications in business competitive environment. Inter. J. Cur. Res. 10(2) 65461-65477

Chatterjee, P; Chakraborty, S. (2012). Material selection using preferential ranking methods. Mat. Design 35: 384-393.

Davood, S; John, E; Rajkumar, R (2005). A review of multicriteria making methods for enhanced maintenance delivery. Procedia CIRP 37, 30-35.

Diakoulaki, D; Mavrotas, G; Papayannakis, L. (1995). Determining Objective Weights in Multiple Criteria Problems: The Critic Method. Computer \& Operation Research 22:763-770.

Diakoulaki, D; Mavrotas, G; Lefteris, P. (1995). Determining objective weights in multiple criteria problems: The CRITIC method. Comp. Operate. Res. 22 (7) $763-770$.

Dong, S.J; Jine, S.J; Eui, H.K. (2005). Development of Integrated Materials Database System for Plant Facilities Maintenance and Optimisation. Key Engineering Materials, Switzerland: Trans Technical Publications 297-300: 2681-2686.

Dragan, P; Zeljko, S; Sinisa, S. (2018). A new model for determining weight coefficient of criteria in MCDM models: Full consistency method (FUCOM). Symmetry 10(9), 393

Ginevicius, R; Podvezko, V. (2005). Objective and subjective approaches to determining the criterion weight in multicriteria models. Proceedings of International Conference RelStat. Transport and Telecommunication 6(1) 133-137.

Jahanshahloo, G.R; Lotfi, F. H; Izadikhah, M. (2006). An algorithmic method to extend TOPSIS for decisionmaking problems with interval data, Applied Mathematics and Computation 175.2: 1375-1384

Jian, M; Zhi-Ping, F; Li-Hua, H. (1999). A subjective and objective integrated approach to determine attribute weights. European Journal of Operational Research, 112(2), 397-404.

Liu, W; Zeng, L (2008). A new TOPSIS method for fuzzy multiple attribute group decision making problem, $J$. Guilin Univ. Electro. Technol. 28.1: 59-62.

Mirko, S; Edmundas, K.Z; Dragan, P; Zeljko, S; Abbas, M. (2019). Application of MCDM methods in sustainability engineering: A literature review, Symmetry 2008-2018.

Olson, L.D. (2008). Subjectivity in Multiple Criteria Decision Analysis. Human Centered Processes.

Parnell, G. and Tainor, T. (2009). Using the Swing Weight to Weight Multiple Objective. Proceeding of the INCOSE international Symposium, Singapore.

Patel, R.M; Vashi, P.M; Bhatt, V.B. (2017). SMART- Multicriteria decision making technique for use in planning activities. NHCE.

Rao, R.V; Davim, J.P. (2008). A decision making framework model for material selection using a combined multiple attribute decision making method. Intern. J. Adv. Technol. 35: 751-760.

Rao, R.V; Patel, B.K. (2011). Material Selection Using a Novel Multiple Attribute Decision Making Method. Inter. J. Man. Mat. Mech. Engineer. 1.1: 43-56.

Roszkowska, W. (2013). Rank Ordering Criteria Weighting Methods-A Comparative Overview. Optimum Studia Ekonomiczne NR, 5(65).

Saaty, T.L. (1977). A scaling method for priorities in heirachical structures. J. Math. Psychology 15(3) 234281.

Saaty, T.L. (1980). The Analytic Hierarchy Process: Planning, Priority Setting, Resource Allocation, McGraw-Hill.

Setiawan, A; Sediyono, E; Moekoe, D.A.L (2014). Application of AHP method in determining priorities of conversion of unused land to food land in Minahasa Tenggara. International Journal of Computer Applications 89(8), 37-44.

Starr, M.K, Greenwood, L.H (1977). Normative generation of alternatives with multiple criteria evaluation. Multiple criteria decision making. Starr M.K, Zeleny M, Eds.. New York, North-Holland. 111-112.

Vinodh, S; Prasanna, M; Praskash, N. H. (2014). Integrated Fuzzy AHP-TOPSIS for selecting the best plastic recycling methods. A case study. Applied Mathematics Modeling, 38(19, 20):4662-4672.

Zardari, N.H; Ahmed, K; Shirazi, S.M; Yusop, Z.B. (2005). Weighting methods and their effects on multi-criteria decision making models outcomes in water resources management. Springer Briefs in Water Sciences and Technology.

$\mathrm{Xu}$, Z. (2007). Methods for aggregating interval-valued intuitionistic fuzzy information and their application to decision making. Control and Decision 22.2: 215-219. 